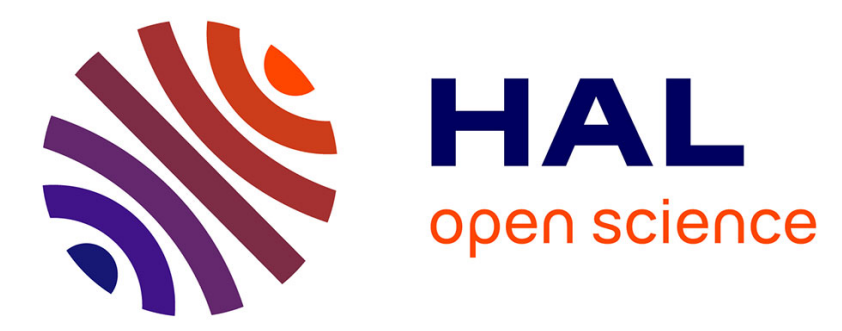

\title{
Gain Scheduling Control of a Nonlinear Electrostatic Microgripper: Design by an Eigenstructure Assignment With an Observer-Based Structure.
}

\author{
Mokrane Boudaoud, Yann Gorrec, Yassine Haddab, Philippe Lutz
}

\section{To cite this version:}

Mokrane Boudaoud, Yann Gorrec, Yassine Haddab, Philippe Lutz. Gain Scheduling Control of a Nonlinear Electrostatic Microgripper: Design by an Eigenstructure Assignment With an ObserverBased Structure.. IEEE Transactions on Control Systems Technology, 2014, pp.1-13. hal-01324599

\author{
HAL Id: hal-01324599 \\ https://hal.science/hal-01324599
}

Submitted on 1 Jun 2016

HAL is a multi-disciplinary open access archive for the deposit and dissemination of scientific research documents, whether they are published or not. The documents may come from teaching and research institutions in France or abroad, or from public or private research centers.
L'archive ouverte pluridisciplinaire $\mathbf{H A L}$, est destinée au dépôt et à la diffusion de documents scientifiques de niveau recherche, publiés ou non, émanant des établissements d'enseignement et de recherche français ou étrangers, des laboratoires publics ou privés. 


\title{
Gain Scheduling Control of a Nonlinear Electrostatic Microgripper: Design by an Eigenstructure Assignment With an Observer-Based Structure
}

\author{
Mokrane Boudaoud, Yann Le Gorrec, Member, IEEE, Yassine Haddab, and Philippe Lutz, Member, IEEE
}

\begin{abstract}
This paper deals with the modeling and the robust control of a nonlinear electrostatic microgripper dedicated to embedded microrobotics applications. We first propose a polynomial linear parameter varying model of the system, where the varying parameter is the mean position of the microgripper that is used for the linearization. The controller is then derived using a multimodel and scheduled observer-based control strategy. The structure and the order of the controller are defined a priori allowing the derivation of a robust low-order controller suitable for a real-time implementation in embedded on-chip environments. Results show that a very wide (several tens of micrometers) and fast positioning of the gripping arm can be achieved using the control strategy. A robustness analysis and experimental implementation results show the efficiency of the controller and the relevance of the theoretical approach.
\end{abstract}

Index Terms-Eigenstructure assignment (ESA), microelectromechanical systems (MEMS), microgripper, multimodel, observer-based structure, robustness, worst case analysis.

\section{NOMENCLATURE}
Modeling
$L, l$, and $e$
Length, width, and thickness of the actuated arm.
$L_{s}, l_{s}$, and $e_{s}$
Half length, width, and thickness of the
double-clamped beams.
$E, \rho \quad$ Young's modulus and mass density.
$I_{S} \quad$ Area moment of inertia of the double-clamped beams.
$F_{\text {elec }}, N$
Electrostatic force and inplane tension.
$\delta\left(x-L_{s}\right)$

Manuscript received December 2, 2013; revised July 2, 2014; accepted September 5, 2014. Manuscript received in final form October 4, 2014. This work was supported in part by the French National Project NanoRobust under Grant ANR 2011 NANO 006 and in part by HAMECMOPSYS under Grant ANR 11-BS03-0002. Recommended by Associate Editor G. Cherubini.

M. Boudaoud is with the Institut des Systèmes Intelligents et de Robotique, Université Pierre et Marie Curie, Paris 75005, France (e-mail: mokrane.boudaoud@isir.upmc.fr).

Y. Le Gorrec, Y. Haddab, and P. Lutz are with the Department of Automatic Control and Micro-Mechatronic Systems in Femto-ST Institute, Université de Franche-Comté, Besançon 25030, France, National Engineering Institute in Mechanics and Microtechnologies, Besançon 25000, France, and also with the Université de Technologie de Belfort Montbéliard, Montbéliard 25200, France (e-mail: legorrec@femto-st.fr; yassine.haddab@femto-st.fr; philippe.lutz@femto-st.fr).

Color versions of one or more of the figures in this paper are available online at http://ieeexplore.ieee.org.

Digital Object Identifier 10.1109/TCST.2014.2362725

$N_{a}$
$\varepsilon$
$h_{z}, g$
$V_{\text {in }}$
$D_{a}$
$y_{a}(L), y_{a}\left(x_{\mathrm{ea}}\right)$

$m_{a}, k_{a}(\cdot), d_{a}(\cdot)$

$k_{1 a}, k_{2 a}, k_{3 a}, k_{4 a}, k_{5 a}, k_{6 a}$

$d_{0 a}, d_{1 a}, d_{2 a}, d_{3 a}, d_{4 a}$

Control

$A_{p}, B_{p}$ and $C_{p}$

$T_{d}$

$\lambda_{r 1}$ and $\lambda_{r 2}$

Total number of fingers (movable and fixed) in the comb drive actuator. Permittivity of the dielectric material.

Thickness of comb fingers and gap spacing between two fingers.

Input voltage of the comb drive actuator.

Amplification parameter.

Position of the actuated arm's tip and position of the midpoint of the double-clamped beams in the $y$-axis.

Effective linear mass of the actuation mechanism, nonlinear stiffness, and damping terms. Linear, quadratic, cubic, and higher order stiffness terms. Linear, quadratic, cubic, and higher order damping terms.

$z_{o}$ $\pi_{o}, u_{o}$ and $t_{o}$ $K_{c}=\left[K_{y} K_{z} K_{i}\right]$ $\Delta_{a}, \Delta_{a 0}$ $\Delta_{a M}$ and $\Delta_{a m}$

$U$

$K_{c 0}, K_{c 1}$ and $K_{c 2}$ Coefficients of the polynomial feedback gain matrix.

$\Gamma_{i}=\left(\lambda_{i}, v_{i}, \omega_{i}\right)$ vector, and the output direction of the closed loop system around an operating point. 


\section{INTRODUCTION}

C ONTROL of microelectromechanical systems (MEMS)-based microgrippers is an important issue in microrobotics [1]. For instance, controlling the position of microgripper arms is crucial when dealing with the manipulation of fragile tiny samples such as deoxyribo nucleic acid (DNA) [2], biological cells [3], or carbon nanotubes [4].

Electrostatic comb drive actuators are very common in the development of MEMS-based microgrippers [5]-[7]. They allow a fast positioning of gripping arms over large displacements (several tens of micrometers), they do not produce heating and they have no hysteretic behavior. Nevertheless, comb drive actuators exhibit strong nonlinearities when exceeding few micrometer displacements only [8]. This is very problematic because large displacements of the actuator are often required to allow the gripping arms to come into contact with objects of various sizes [e.g., [9] reported that most animal cells (including human) range in size between 1 and $100 \mu \mathrm{m}]$. These nonlinearities arise from several physical phenomena among which the inplane tension [10], the structural damping [11], the external electrostatic potentials [12], and the side instability [13]. Satisfying robust closed loop performance to comb drive actuators over a wide operating range is very challenging and few studies tackle this problem [14] although it is essential for micromanipulation. Moreover, with the need of performing microrobotics tasks in embedded environments, the controller must have a simple and low-dimensional structure and must not be time consuming to be implemented in simple microcontrollers [15].

In that sense, gain scheduling is an interesting solution to deal with nonlinear problems when the nonlinearities can be derived from measurable and bounded varying parameters [18], [19]. As such, numerous approaches have been developed to extend the traditional robust control strategies dedicated to linear time invariant (LTI) systems to the nonlinear systems case. Such approaches mainly come from the generalization of the $\mathrm{H} \infty$ control design to the control of linear parameter varying (LPV) systems [20]. One of the major drawbacks of $\mathrm{LPV} / \mathrm{H} \infty$ control techniques is that they are conservative and they often lead to high order and time-consuming controllers [21]. The development of both less conservative and low-order gain-scheduled controllers has been for a long time very challenging [23], [22] especially when controllers have to be implemented experimentally [15]. The multimodel approach [24] is an interesting solution to reduce significantly the conservatism of the controller by considering a finite number of LTI models in the set of the varying parameters instead of considering a continuum of models [16]. The multimodel constraints can be defined from a worst case analysis aiming at ensuring the stability and some robust performance [17].

In this paper, we focus on the modeling and the robust control of a class of MEMS-based microgrippers using nonlinear comb drive actuators (see examples in [2] and [5]-[7]). The aim is to propose a systematic methodology (from the modeling to the control) aiming at improving,

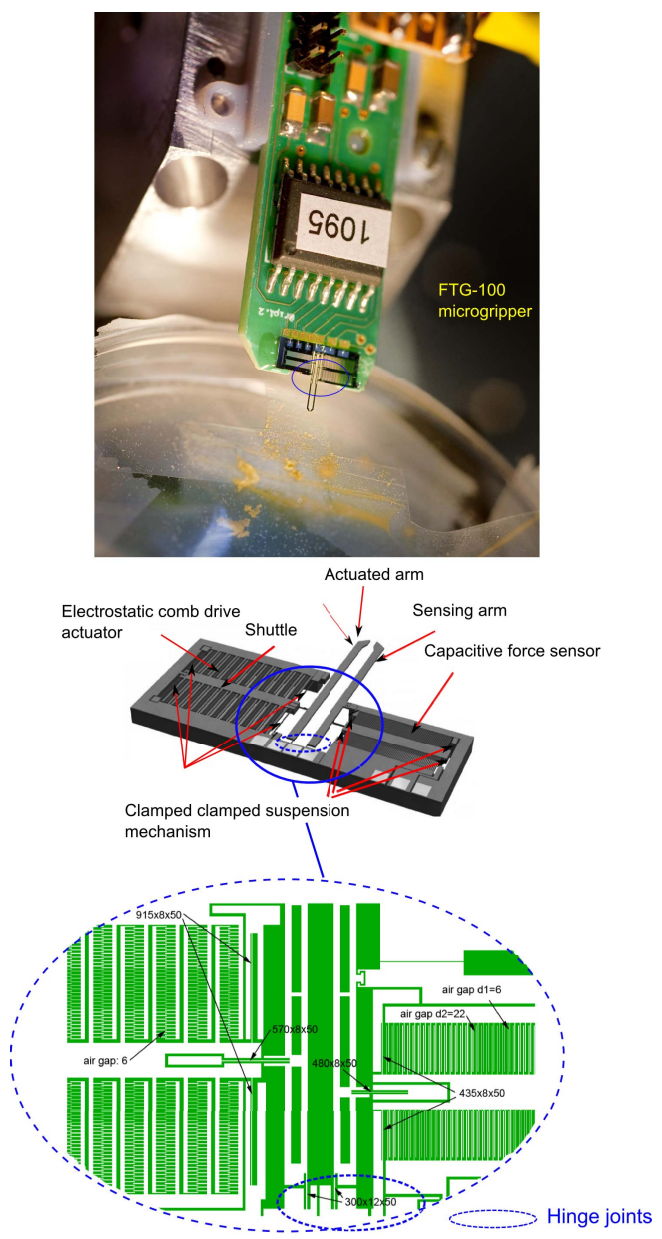

Fig. 1. Structure of the FT-G100 microgripper (FemtoTools GmbH).

using a low-order controller, the performance of this class of microgrippers considering requirements of microrobotics applications. For instance, the ability of performing the manipulation of objects of various sizes (e.g., 1-100 $\mu \mathrm{m}$ ) and the need of achieving very fast pick-and-place operations for industrial applications. A microgripper (FT-G100) from FemtoTools GmbH Company [6] is used as a case of study (Fig. 1). This device is widely used in robotics laboratories but very few control solutions have been developed to increase its efficiency when operating at the microscale.

In this paper, a nonlinear modeling approach of the actuation mechanism is proposed and validated with experiments over a large operating range of the system. The model considers the nonlinear stiffness and the nonlinear damping that are inherent to comb drive actuators. A gain-scheduled controller is thereafter designed to ensure some performance level over a wide operating range despite nonlinearities. Nonlinear parameters, namely the stiffness and the damping experimentally identified, are matched. Both are expressed in term of the actuated arm tip displacement that is measurable and can be used as scheduling parameter. Therefore, instead of using the nonlinear parameters independently as scheduling variables, we propose to use only one scheduling parameter. To do so, a polynomial LPV model of the system is introduced.

The control strategy is based on a multimodel eigenstructure assignment (ESA) and uses an observer-based 

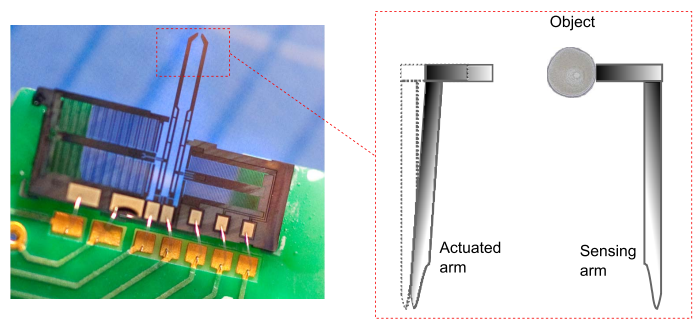

Fig. 2. Initial configuration of gripping arms at Step 2. Dashed line: initial position of the actuated arm.

structure. To deal with very large parameter variations, both the controller and the observer are scheduled. The structure of the controller (namely an output feedback gain) with respect to the scheduling variable is polynomial. The order of the polynomial is defined a priori which allows the user to set the dimension of the controller considering real-time implementation constraints. This approach is advantageous comparing with classic gain-scheduled controllers [19] which are interpolated a posteriori with respect to scheduling variables. The relevance of the control approach is demonstrated by considering the FT-G100 microgrippers, and experimental results show that a very large and fast positioning of the gripping arm can be achieved in closed loop by using a very simple and low-dimensional (i.e., low-order) controller which is of a great importance from the application point of view. Such a result leads to new perspectives for high precision and fast micromanipulation tasks in embedded environments.

This paper is organized as follows. In Section II, the global architecture of the microgripper and its working principle are presented. Sections III and IV deal with the nonlinear modeling and the LPV modeling of the actuation mechanism. The closed loop control strategy is presented in Section V. In Section VI, a robustness analysis and experimental control results are presented. Section VII includes a discussion on real-time implementation issues.

\section{Working PrinciPle Of THE MiCROGRIPPER}

The FT-G100 microgripper features two main parts (Fig. 1): 1) an actuation mechanism composed of a comb drive actuator and an actuated arm and 2) a sensing mechanism (for the measurement of the gripping force) including a capacitive sensor and a sensing arm. The initial opening of the gripping arms is $100 \mu \mathrm{m}$ and the full close is achieved when applying $200 \mathrm{~V}$ actuation voltage to the comb drive actuator. When a voltage is applied to the actuator, an electrostatic force is generated allowing the motion of a silicon structure (shuttle) connected to the actuated arm through a slender beam. The base of the actuated arm is composed of a pseudorigid beam. A suspension mechanism composed of two pairs of doubleclamped beams hold the shuttle to produce a restoring force.

The manipulation of an object with a two fingered microrobotic system requires three steps.

Step 1: The carrier (i.e., serial robotic system or a micropositioning table) of the microgripper must allow positioning the micromanipulation system such that the object to be manipulated is in contact only with the sensing arm (Fig. 2).

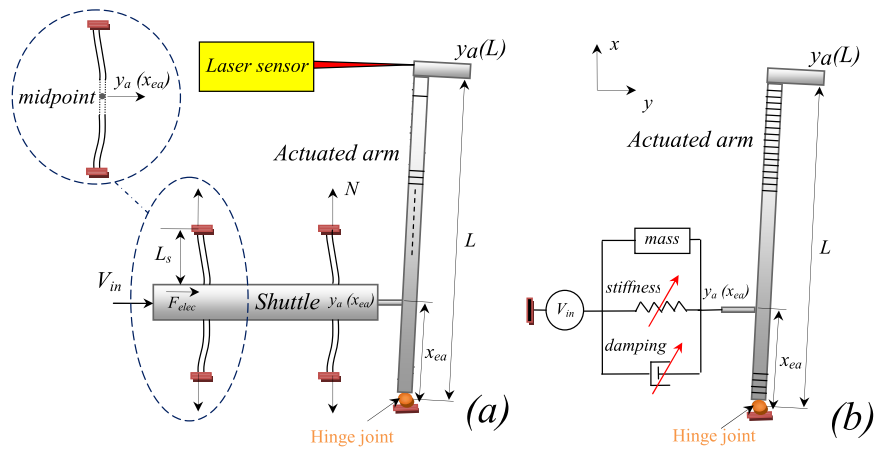

Fig. 3. (a) Simplified scheme of the actuation mechanism. (b) Equivalent scheme of suspensions.

TABLE I

Dimensions of the Actuation Mechanism

\begin{tabular}{lll}
\hline & Actuated arm & Doubly-clamped beams \\
\hline Length & $L=5150 \mu \mathrm{m}$ & $L_{s}=915 \mu \mathrm{m}$ (half length) \\
\hline Width & $l=150 \mu \mathrm{m}$ & $l_{s}=50 \mu \mathrm{m}$ \\
\hline Thickness & $e=50 \mu \mathrm{m}$ & $e_{s}=8 \mu \mathrm{m}$ \\
\hline
\end{tabular}

Step 2: The tip position of the actuated arm has to be controlled in closed loop to allow it coming in contact with the object.

Step 3: When both the tips of the actuated and sensing arms are in contact with the object, the gripping force has to be controlled. In this paper, we tackle the issue of Step 2.

\section{Nonlinear Modeling AND IDENTIFICATION}

In this section, a nonlinear dynamic model of the actuation mechanism is proposed. The model describes the dynamic transfer between the input voltage $V_{\text {in }}$ and the position $y_{a}(L)$. Static and dynamic parameters of the model are identified experimentally and a final nonlinear model is derived for the control.

\section{A. Nonlinear Dynamic Modeling}

The modeling is performed under the following hypothesis (see [25] for hypothesis verification): 1) the actuated arm is rigid in the $y$-axis; 2) the slender beam between the shuttle and the actuated arm is not subject to buckling; and 3) the pseudorigid beam behaves as a hinge joint.

Therefore, we consider a nonlinear Euler Bernoulli equation describing the undamped bending of the suspension mechanism at the midpoint of the double-clamped beams $y_{a}\left(x_{\mathrm{ea}}\right)$ in the $y$-axis [Fig. 3(a)]

$$
\begin{aligned}
E I_{s} \frac{\partial^{4} y_{a}\left(x_{\mathrm{ea}}\right)}{\partial x^{4}}+\rho l_{s} e_{s} \frac{\partial^{2} y_{a}\left(x_{\mathrm{ea}}\right)}{\partial t^{2}}+ & N \frac{\partial^{2} y_{a}\left(x_{\mathrm{ea}}\right)}{\partial x^{2}} \\
& =F_{\mathrm{elec}} \delta\left(x-L_{s}\right)
\end{aligned}
$$

where $E$ is the Young's modulus, $I_{s}$ is the area moment of inertia of the double-clamped beams, $\rho$ is the mass density, $F_{\text {elec }}$ is the electrostatic force, and $\delta$ is the Dirac delta function. Moreover, $L_{s}, l_{s}$, and $e_{s}$ are dimensions as described in Table I. 
The two first terms of (1) relate to the linear Euler Bernoulli beam equation. The third term introduces a nonlinearity because of the so-called inplane tension located at the clamped parts of the suspensions [Fig. 3(a)]. The inplane tension $N$ is given by [10]

$$
N=\frac{E l_{s} e_{s}}{4 L_{s}} \int_{0}^{2 L_{s}}\left(\frac{\partial y_{a}}{\partial x}\right)^{2} \partial x .
$$

The electrostatic force is governed by the following equation [26]:

$$
F_{\text {elec }}=\frac{N_{a} \varepsilon h_{z}}{2 g} \cdot V_{\text {in }}^{2}
$$

where $V_{\text {in }}$ is the actuation voltage, $N_{a}=1300$ is the total number of fingers (movable and fixed) in the actuator, $\varepsilon=8.85 \mathrm{pF} / \mathrm{m}$ is the permittivity of the dielectric material (air), $h_{z}=50 \mu \mathrm{m}$ is the thickness of comb fingers, and $g=6 \mu \mathrm{m}$ is the gap spacing between two fingers.

Considering the hypothesis of the modeling, the variable $y_{a}(L)$ is introduced in (1) leading to

$$
\begin{aligned}
\frac{E I_{s}}{D_{a}} \frac{\partial^{4} y_{a}(L)}{\partial x^{4}}+\frac{\rho l_{s} e_{s}}{D_{a}} \frac{\partial^{2} y_{a}(L)}{\partial t^{2}}+\frac{N}{D_{a}} \frac{{\frac{\partial}{2} y_{a}(L)}_{\partial x^{2}}}{}=F_{\mathrm{elec}} \delta\left(x-L_{s}\right)
\end{aligned}
$$

where $D_{a}=L / x_{\text {ea }}$ is an amplification parameter $\left(x_{\mathrm{ea}}=\right.$ $1100 \mu \mathrm{m})$.

Substituting (2) into (4) gives the partial differential equation

$$
\begin{aligned}
& \frac{E I_{s}}{D_{a}} \frac{\partial^{4} y_{a}(L)}{\partial x^{4}}+\frac{\rho l_{s} e_{s}}{D_{a}} \frac{\partial^{2} y_{a}(L)}{\partial t^{2}} \\
& \quad+\left[\frac{E l_{s} e_{s}}{4 L_{s} D_{a}} \int_{0}^{2 L_{s}}\left(\frac{\partial y_{a}}{\partial x}\right)^{2} \partial x\right] \frac{\partial^{2} y_{a}(L)}{\partial x^{2}}=F_{\mathrm{elec}} \delta\left(x-L_{s}\right) .
\end{aligned}
$$

Thus, using the perturbation method (see [10] for more information), (5) can be simplified into a lumped parameter equation of the system.

Such an equation is given by

$$
m_{a_{0}} \frac{\partial^{2} y_{a}(L)}{\partial t^{2}}+k_{1 a} y_{a}(L)+k_{3 a} y_{a}^{3}(L)=\frac{1}{D_{a}} F_{\text {elec }}
$$

with

$$
\begin{aligned}
& m_{a_{0}}=\frac{\rho l_{s} e_{s}}{\phi_{1}^{2}\left(2 L_{s}\right) D_{a}^{2}} \int_{0}^{2 L_{s}} \phi_{1}^{2}(x) \cdot \partial x \\
& k_{1 a}=\frac{2 E I_{s}}{\phi_{1}^{2}\left(2 L_{s}\right) D_{a}^{2}} \int_{0}^{2 L_{s}}\left(\phi_{1}^{\prime \prime}(x)\right)^{2} \cdot \partial x \\
& k_{3 a}=\frac{E}{\phi_{1}^{2}\left(2 L_{s}\right) D_{a}^{2}}\left(\int_{0}^{2 L_{s}}\left(\phi_{1}^{\prime}(x)\right)^{2} \cdot \partial x\right)^{2}
\end{aligned}
$$

where $\phi_{1}$ is the shape mode of the first Eigen mode of the suspensions.

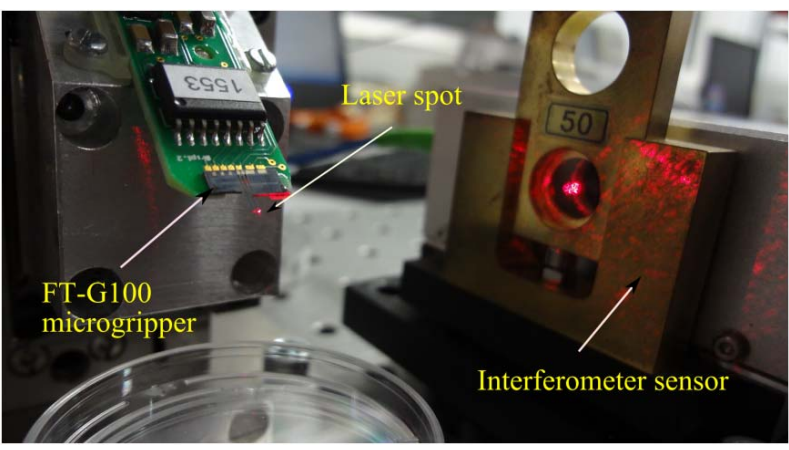

Fig. 4. Measurement of the actuated arm position by using a laser interferometer sensor.

In order to account the mass of the shuttle and that of the actuated arm in (6), the term $M_{\mathrm{sh}}$ is introduced. Moreover, a nonlinear term $d_{a}(\cdot)$ is introduced to to account for the variations of the damping with increasing the actuation voltage. The structure (model) of this term is given in the sequel. Hence, (6) becomes

$m_{a} \frac{\partial^{2} y_{a}(L)}{\partial t^{2}}+d_{a}(\cdot) \frac{\partial y_{a}(L)}{\partial t}+k_{1 a} y_{a}(L)+k_{3 a} y_{a}^{3}(L)=\frac{1}{D_{a}} F_{\text {elec }}$

where $m_{a}=m_{a_{0}}+M_{\mathrm{sh}}$ and $k_{1 a}$ are respectively the effective linear mass and the effective linear stiffness of the actuation mechanism. The nonlinear term $k_{3 a}$ (called cubic stiffness) is due to the inplane tension $N$.

In the next section, both static and dynamic parameters of the nonlinear model are identified using experimental data from the commercial FT-G100 microgripper.

\section{B. Identification}

A high-resolution $(0.01 \mathrm{~nm})$ laser interferometer (SP-120 SIOS meßtechnik $\mathrm{GmbH}$ ) is used to perform measurements of the displacement $y_{a}(L)$ at the tip of the actuated arm (Fig. 4). Input voltages are generated using a dSPACE control board (DS1103). First, $k_{1 a}$ and $k_{3 a}$ are identified through the experimental static characteristic $y_{a}(L) / V_{\text {in }}$ for $0<V_{\text {in }}<$ $200 \mathrm{~V}$. Experimental data are then fitted using (7) in static mode (i.e., for $t \rightarrow \infty$ ). As a result, the mean error of the static characteristic in the whole operating range of the system is found to be equal to $17.11 \%$.

In [27], it is commonly admitted that in addition to the inplane tension, other sources of nonlinearities such electrostatic nonlinearities can produce nonlinear stiffness terms of higher orders. Therefore, to reduce the mean fitting error to less than $10 \%$, the nonlinear stiffness is described by a sixth order polynomial leading to the following expression:

$$
k_{a}(\cdot)=\sum_{i=1}^{6} k_{i a} y_{a}^{i-1}(L) .
$$

As such, in static mode, (7) becomes

$$
\begin{aligned}
& k_{1 a} y_{a}(L)+k_{2 a} y_{a}^{2}(L)+k_{3 a} y_{a}^{3}(L)+k_{4 a} y_{a}^{4}(L) \\
& +k_{5 a} y_{a}^{5}(L)+k_{6 a} y_{a}^{6}(L)=\frac{1}{D_{a}} F_{\text {elec }} .
\end{aligned}
$$



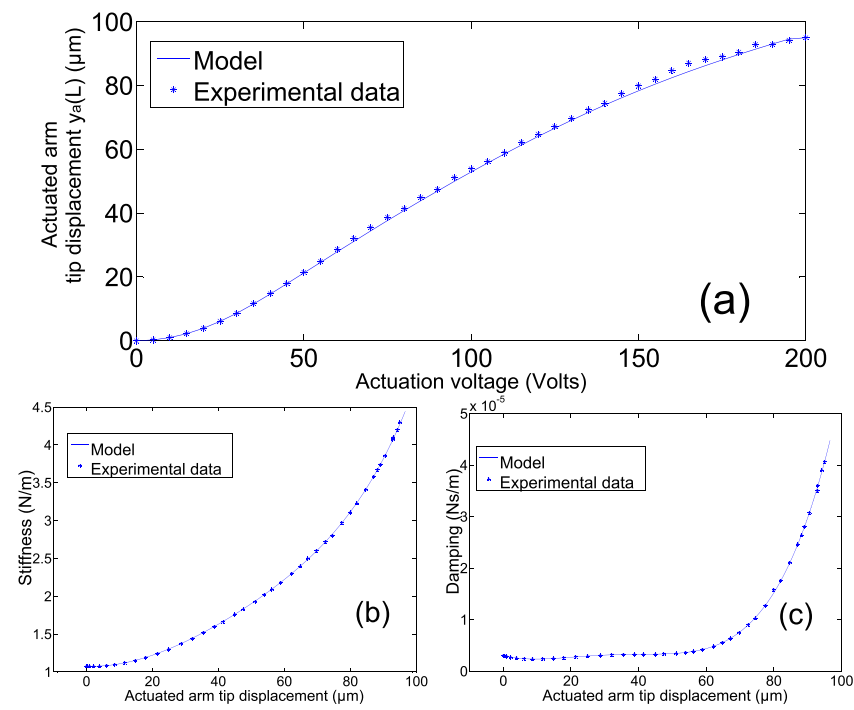

Fig. 5. Nonlinear characteristics of the FT-G100 actuation mechanism. (a) $y_{a}(L) / V_{\text {in }}$. (b) $k_{a}(\cdot) / y_{a}(L)$. (c) $d_{a}(\cdot) / y_{a}(L)$.

TABLE II

IDENTIFIED STATIC AND DYNAMIC PARAMETERS of the ACtUation Mechanism

\begin{tabular}{lll}
\hline Symbol & Value & Description \\
\hline \hline$m_{a}$ & $3.9843 \times 10^{-8} \mathrm{~kg}$ & mass \\
\hline$k_{1 a}$ & $1.0762 \mathrm{~N} / \mathrm{m}$ & stiffness terms \\
$k_{2 a}$ & $-1.6 \times 10^{3} \mathrm{~N} / \mathrm{m}^{2}$ & \\
$k_{3 a}$ & $4.88 \times 10^{8} \mathrm{~N} / \mathrm{m}^{3}$ & \\
$k_{4 a}$ & $-1.69 \times 10^{12} \mathrm{~N} / \mathrm{m}^{4}$ & \\
$k_{5 a}$ & $-3.98 \times 10^{16} \mathrm{~N} / \mathrm{m}^{5}$ & \\
$k_{6 a}$ & $4.71 \times 10^{20} \mathrm{~N} / \mathrm{m}^{6}$ & \\
\hline$d_{0 a}$ & $3.01 \times 10^{-6} \mathrm{Ns} / \mathrm{m}$ & damping terms \\
$d_{1 a}$ & $-0.162 \mathrm{Ns} / \mathrm{m}^{2}$ & \\
$d_{2 a}$ & $1.24 \times 10^{4} \mathrm{Ns} / \mathrm{m}^{3}$ & \\
$d_{3 a}$ & $-3.04 \times 10^{8} \mathrm{Ns} / \mathrm{m}^{4}$ & \\
$d_{4 a}$ & $2.47 \times 10^{12} \mathrm{Ns} / \mathrm{m}^{5}$ & \\
\hline
\end{tabular}

The mean fitting error is reduced to $3.66 \%$ and results are shown in Fig. 5(a). The identified linear, quadratic, cubic, and higher order stiffness terms are given in Table II. Consequently, the nonlinear characteristic of the stiffness is deduced as shown in Fig. 5(b).

Thereafter, step voltages are applied to the actuator with amplitudes ranging from 5 to $200 \mathrm{~V}$ (40 steps excitation are applied). For each excitation step, the response $y_{a}(L)$ is measured experimentally. The mass is identified from the step response corresponding to $5 \mathrm{~V}$ step excitation and the damping is identified at each operating point (from 5 to $200 \mathrm{~V}$ ). Results show that the damping increases with increasing the amplitude of the step excitation starting from $y_{a}(L)=60 \mu \mathrm{m}$ [Fig. 5(c)].

To describe accurately the nonlinear damping of the system using experimental identification results, a fourth order polynomial of the form

$$
d_{a}(\cdot)=\sum_{i=0}^{4} d_{i a} y_{a}^{i}(L)
$$
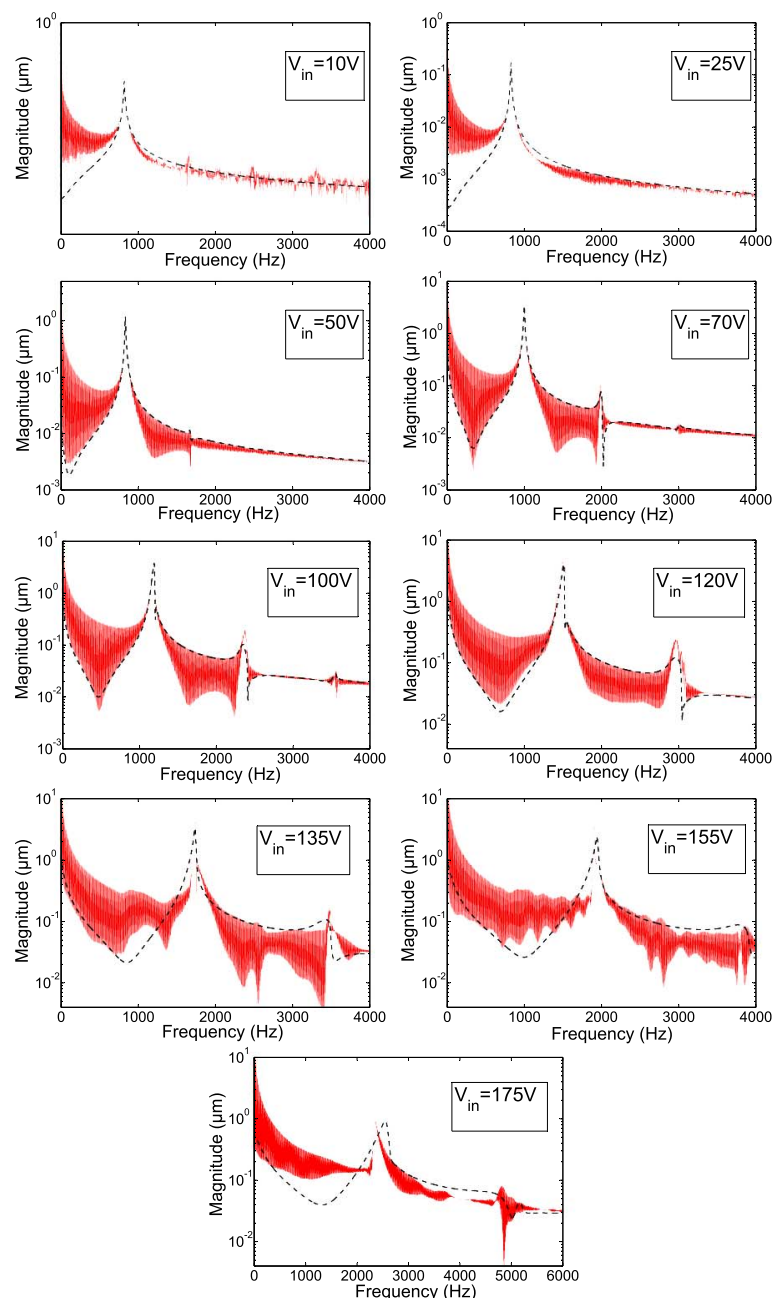

Fig. 6. Frequency responses of the nonlinear actuation mechanism. Continuous line: experimental data. Dashed line: simulation data.

has been used. Taking into account the nonlinear terms of the stiffness and the damping, the nonlinear state space model of the actuation mechanism is given as

$$
\left\{\begin{aligned}
& {\left[\begin{array}{l}
\dot{y}_{a}(L) \\
\ddot{y}_{a}(L)
\end{array}\right]=} {\left[\begin{array}{cc}
0 & 1 \\
-\sum_{i=1}^{6} \frac{k_{i a}}{m_{a}} y_{a}^{i-1}(L)-\sum_{i=0}^{4} \frac{d_{i a}}{m_{a}} y_{a}^{i}(L)
\end{array}\right]\left[\begin{array}{l}
y_{a}(L) \\
\dot{y}_{a}(L)
\end{array}\right] } \\
&+\left[\begin{array}{c}
0 \\
\frac{K_{e}}{m_{a} D_{a}}
\end{array}\right] \cdot V_{\mathrm{in}}^{2} \\
& y_{a}(L)=\left[\begin{array}{ll}
1 & 0
\end{array}\right]\left[\begin{array}{l}
y_{a}(L) \\
\dot{y}_{a}(L)
\end{array}\right] \\
& K_{e}=\frac{N_{a} \varepsilon h_{z}}{2 g} .
\end{aligned}\right.
$$

To validate the modeling approach based on nonlinear polynomial terms, frequency responses of the model are compared with experimental ones. For each operating point, experimental and simulated step responses are recorded and a fast Fourier transform is computed. Results are shown in Fig. 6.

The nonlinear model describes accurately the increase of the fundamental resonance frequency with increasing the input 
voltage owing to the nonlinear stiffness. The fundamental resonance frequency increases from $827 \mathrm{~Hz}$ to more than $2 \mathrm{kHz}$ for $5 \mathrm{~V}<V_{\text {in }}<175 \mathrm{~V}$. For $V_{\text {in }}>70 \mathrm{~V}$, higher order eigenmodes can be observed experimentally. The model is able to capture this characteristic.

For $V_{\text {in }}>155 \mathrm{~V}$, the value of the fundamental resonance frequency obtained by simulation does not agree with experiments, because the actuated arm is close to the sensing arm. Hence, the overshoot of the step response causes a contact between the two arms. This contact is not considered in the model.

The polynomial structure of nonlinear stiffness and damping terms is one of the main characteristics of comb drive actuators (see [27] for more details). As shown in Fig. 6, the model is sufficiently accurate and can be therefore used for the synthesis of the controller and for the robustness analysis.

\section{LPV Modeling AND CONTROL SPECIFICATIONS}

\section{A. Polynomial LPV Model}

To design a gain-scheduled controller, a LPV model is derived from the nonlinear model (11). The nonlinear model can be reformulated into an affine (i.e., linear) LPV model, if the stiffness $k_{a}(\cdot)$ and the damping $d_{a}(\cdot)$ [see (8) and (10)] are selected as independent varying parameters for which only their lower and their upper bounds are considered. In this case, well-known gain-scheduled control strategies based on the $\mathrm{LPV} / \mathrm{H} \infty$ methodology can be used (see an example in [21]).

In the case of comb drive actuators, the nonlinear parameters $k_{a}(\cdot)$ and $d_{a}(\cdot)$ are matched and they have a polynomial structure. Such properties are usually not taken into account with a classical affine $\mathrm{LPV} / \mathrm{H} \infty$ design which is very conservative. In this paper, the use of the operating point (position of the actuated arm tip) $\Delta_{a}$ as the only varying parameter is proposed. As such, using a Jacobian linearization, the nonlinear plant (11) is formulated into a polynomial LPV model of the form

$$
\begin{aligned}
G_{a}\left(s, \Delta_{a}\right): & \left\{\begin{array}{l}
\dot{X}_{p}(t)=A_{p}\left(\Delta_{a}\right) X_{p}(t)+B_{p} U(t) \\
\tilde{y}_{a}(L)=C_{p} X_{p}(t)
\end{array}\right. \\
A_{p}\left(\Delta_{a}\right)= & {\left[\begin{array}{cc}
0 & 1 \\
\frac{-k_{1 a}}{m_{a}} & \frac{-d_{0 a}}{m_{a}}
\end{array}\right]+\Delta_{a}\left[\begin{array}{cc}
0 & 1 \\
\frac{-2 k_{2 a}}{m_{a}} & \frac{-d_{1 a}}{m_{a}}
\end{array}\right]+\cdots } \\
& +\Delta_{a}^{2}\left[\begin{array}{cc}
0 & 1 \\
\frac{-3 k_{3 a}}{m_{a}} & \frac{-d_{2 a}}{m_{a}}
\end{array}\right]+\Delta_{a}^{3}\left[\begin{array}{cc}
0 & 1 \\
\frac{-4 k_{4 a}}{m_{a}} & \frac{-d_{3 a}}{m_{a}}
\end{array}\right]+\cdots \\
& +\Delta_{a}^{4}\left[\begin{array}{cc}
0 & 1 \\
\frac{-5 k_{5 a}}{m_{a}} & \frac{-d_{4 a}}{m_{a}}
\end{array}\right]+\Delta_{a}^{5}\left[\begin{array}{cc}
0 & 1 \\
\frac{-6 k_{6 a}}{m_{a}} & 0
\end{array}\right] \\
B_{p}= & {\left[\begin{array}{c}
0 \\
\frac{K_{e}}{m_{a} D_{a}}
\end{array}\right], C_{p}=\left[\begin{array}{c}
1 \\
0
\end{array}\right]^{T}, X_{p}=\left[\begin{array}{l}
y_{a}(L) \\
\dot{y}_{a}(L)
\end{array}\right] }
\end{aligned}
$$

where $A_{p} \in \mathbb{R}^{n_{a} \times n_{a}}, B_{p} \in \mathbb{R}^{n_{a} \times m}$ and $C_{p} \in \mathbb{R}^{p \times n_{a}}$, with $n_{a}=2, m=1$, and $p=1$. $\tilde{y}_{a}(L)$ is the variation of $y_{a}(L)$ around the operating point $\Delta_{a}$.

To simplify notations, the variable $\tilde{y}_{a}(L)$ is denoted $y_{a}(L)$ in what follows. The nonlinearity arising from the square voltage is overcome by considering $U=V_{\text {in }}^{2}$ as the input of the model for the controller design. This consideration is valid because

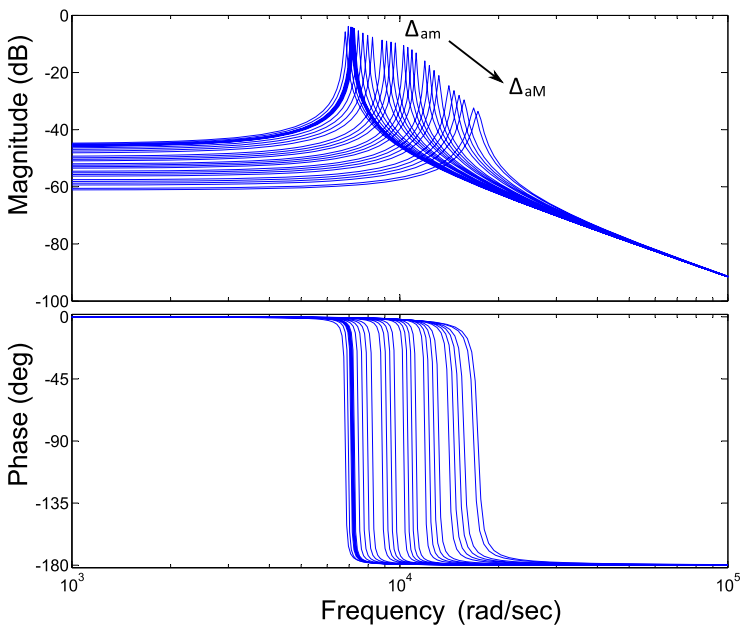

Fig. 7. Bode diagram of elementary linear models for $\Delta_{a m}<\Delta_{a}<\Delta_{a M}$.

only positive values of the input voltage are applied to the actuator. Let us consider $\Delta_{a m}$ and $\Delta_{a M}$ as the operating points of the actuation mechanism corresponding to $V_{\text {in }}=5 \mathrm{~V}$ and $V_{\text {in }}=175 \mathrm{~V}$, respectively. For $\Delta_{a m}<\Delta_{a}<\Delta_{a M}$, Bode diagram of the elementary linear models obtained from (12) are shown in Fig. 7.

Control specifications required in this paper are derived from general need in micromanipulation in terms of accuracy, closed loop bandwidth, and vibration damping.

For nominal performance, control specifications are given as follows.

1) The closed loop response time of the system must be lower than $15 \mathrm{~ms}$ (ten times smaller than the response time of the open loop system). To our best knowledge, such a fast response time has never been achieved and never been demonstrated experimentally using a MEMSbased electrostatic microgripper. It is of a great importance for a high speed and a reliable characterization and assembly of nanostructures.

2) No overshoot is admitted. An overshoot of few micrometers can destroy a manipulated object.

3) The maximum static error must be lower than $1 \%$. Considering the nominal performance, a reference closed loop transfer function $T_{d}$ is defined as

$$
T_{d}=\frac{0.9995}{4 \times 10^{-6} s^{2}+0.006 \mathrm{~s}+1} \text {. }
$$

The reference model $T_{d}$ contains two eigenvalues $\lambda_{1}=-191$ and $\lambda_{2}=-1300$.

\section{GAIN-SCheduled CONTROL}

The control strategy is based on an observer-based multimodel ESA [28], [29] approach. The observer is designed to increase artificially the number of outputs that can be used for control purposes. Both the controller and the observer are scheduled using the measurable mean position of the tip. The scheduling of the observer guarantees the separation principle for all the system configurations and the scheduling of the controller allows a design through a multimodel technique 
accounting for the overall system closed loop performances. The structure of the output feedback gain with respect to the scheduling variable $\Delta_{a}$ is a priori defined leading to the resolution of a simple set of linear equations.

In the sequel, the control strategy (observer+static feedback) is first derived considering a nominal configuration, that is, on a model configuration corresponding to a given $\Delta_{a}$ (that is frozen). Then the variations of $\Delta_{a}$ are taken into account through an iterative procedure altering multimodel ESA and worst case analysis.

\section{A. ESA Considering a Frozen Value $\Delta_{a}=\Delta_{a}$}

First, let us recall the classical ESA method [30] where $A_{p}, B_{p}$, and $C_{p}$ are the system with frozen parameter, that is, $\Delta_{a}=\Delta_{a 0}$.

Lemma 1: Given the closed loop eigenvalue $\lambda_{i}$, the triple $\Gamma=\left(\lambda_{i}, v_{i}, \omega_{i}\right)$ satisfying

$$
\left[A_{p}-\lambda_{i} I_{n}-B_{p}\right]\left[\begin{array}{c}
v_{i} \\
\omega_{i}
\end{array}\right]=0
$$

is assigned by the static gain $K_{c}$ if and only if

$$
K_{c} C_{p} v_{i}=\omega_{i}
$$

where $v_{i} \in \mathbb{C}^{n}$ and $\omega_{i} \in \mathbb{C}^{m}$ are, respectively, the eigenvector and the input direction of the closed loop system.

This control strategy has two main limitations: 1) the degrees of freedom of the controller (i.e., number of triples that can be assigned in closed loop) is limited by the number of output of the system and 2) the controller is generally not robust against the parametric variations of the system.

The system having two states and one output, it is necessary to increase artificially this number to solve (15).

As such, considering the reference model (13), to assign the eigenvalues to the frozen parameter system (12), we propose to add an observer-based structure by using Proposition 2 [28].

Proposition 1: The system defined by (Fig. 9)

$$
\begin{aligned}
\frac{d z_{o}}{d t} & =\pi_{o} z_{o}-t_{o} y_{a}(L)+u_{o} B_{p} U \\
\pi_{o} u_{o} & =u_{o} A_{p}\left(\Delta_{a 0}\right)+t_{o} C_{p}
\end{aligned}
$$

where $u_{o} \in \mathbb{C}^{n}, t_{o} \in \mathbb{C}^{p}$ and $\pi_{o} \in \mathbb{C}$ is an observer of the variable $z_{o}=u_{o} X_{p}$ and the observation error $\varepsilon_{o}=z_{o}-u_{o} X_{p}$ satisfies $\partial \varepsilon_{o} / \partial t=\pi_{o} \varepsilon_{o}$.

This Lemma highlights that a linear combination of the states $u_{o} X_{p}$ can be estimated by an observer increasing the number of output that can be used by the static feedback. It then allows assigning as many more triples than the number of observations.

Therefore, the control problem consists now in finding a gain matrix $K_{c}=\left[K_{y} K_{z}\right]$ such that the system

$$
\left\{\begin{array}{l}
\dot{X}_{p}=A_{p}\left(\Delta_{a 0}\right) X_{p}+B_{p} U \\
\frac{d z_{o}}{d t}=\pi_{o} z_{o}-t_{o} y_{a}(L)+u_{o} B_{p} U \\
y_{a}(L)=C_{p} X_{p}
\end{array}\right.
$$

controlled by the input

$$
U=-K_{y} y_{a}(L)-K_{z} z_{o}
$$

has the expected performances.

In the sequel, we consider the control problem by considering the separation principle described in Theorem 1.

Theorem 1: It is equivalent to assign with the static gain $K_{c}=\left[\begin{array}{ll}K_{y} & K_{z}\end{array}\right]$ the eigenvalues of the system (18) and that of the system

$$
\left\{\begin{array}{l}
\dot{X}_{p}=A_{p}\left(\Delta_{a 0}\right) X_{p}+B_{p} U \\
{\left[\begin{array}{l}
y_{a}(L) \\
z_{o}
\end{array}\right]=\left[\begin{array}{l}
C_{p} \\
u_{o}
\end{array}\right] X_{p} .}
\end{array}\right.
$$

The structure of model (20) allows defining explicitly the variables $y_{a}(L)$ and $z_{o}$ as the output of the system.

Moreover, to satisfy the closed loop precision requirements, model (20) is augmented by an integrator. As such, the control law is now defined by

$$
U=K_{i} \int_{0}^{t}\left(y_{c}-y_{a}(L)\right) d t-K_{y} y_{a}(L)-K_{z} z_{o}
$$

where $K_{i}$ is the gain of the integrator. The controller's gain becomes $K_{c}=\left[\begin{array}{lll}K_{y} & K_{z} & K_{i}\end{array}\right]$.

The order of the open loop system is now equal to three, and the three measurements available are: the output of the system $y_{a}$, the output of the observer $z_{o}$, and the signal $y_{c}-y_{a}$.

\section{B. ESA Considering the Variation of $\Delta_{a}$}

In this section, the design of both the scheduled observer and the scheduled controller's gain $K_{c}$ are described. The scheduled controller is designed through a multimodel approach.

1) Design of the Scheduled Observer: We consider now the variation of $\Delta_{a}$ in a set $\left[\Delta_{a m} \Delta_{a M}\right.$ ]. In such a case, the separation principle (Theorem 1) is no longer satisfied $\forall \Delta_{a} \in$ $\left[\begin{array}{ll}\Delta_{a m} & \Delta_{a M}\end{array}\right]$. Indeed, the observer depends on $\Delta_{a 0}$ and (17) is not satisfied for all $\Delta_{a}$. To account for this drawback, one can modify the observer structure using a scheduling strategy.

The condition (17) can be written under the following form:

$$
\left[\begin{array}{ll}
u_{o} & t_{o}
\end{array}\right]\left[\begin{array}{c}
A_{p}\left(\Delta_{a}\right)-\pi_{o} I_{n} \\
C_{p}
\end{array}\right]=0
$$

where $\Delta_{a} \in\left[\begin{array}{ll}\Delta_{a m} & \Delta_{a M}\end{array}\right]$.

Hence, if $\pi_{o}$ is different from any eigenvalue of the matrix $A_{p}\left(\Delta_{a}\right)$, and $t_{0}$ a priori chosen, the relation (22) is equivalent to

$$
u_{o}\left(\Delta_{a}\right)=t_{o} C_{p}\left(\pi_{o} I_{n}-A_{p}\left(\Delta_{a}\right)\right)^{-1} .
$$

Observer shown in Fig. 8 with $u_{o}$ satisfying (23) is a scheduled formulation of the observer. It allows satisfying the separation principle $\forall \Delta_{a} \in\left[\begin{array}{ll}\Delta_{a m} & \Delta_{a M}\end{array}\right]$.

2) Design of the Scheduled Gain-Multimodel Approach: To guarantee the performances in the entire set $\left[\begin{array}{ll}\Delta_{a m} & \Delta_{a M}\end{array}\right]$, we propose to design the gain $K_{c}=\left[K_{y}, K_{z}, K_{i}\right]$ through a multimodel approach. The output feedback $K_{c}$ will follow the variation of $\Delta_{a}$ through a predefined law. Here, we define a second-order polynomial law with respect to $\Delta_{a}$ as follows:

$$
K_{c}\left(\Delta_{a}\right)=K_{c 0}+K_{c 1} \Delta_{a}+K_{c 2} \Delta_{a}^{2}
$$




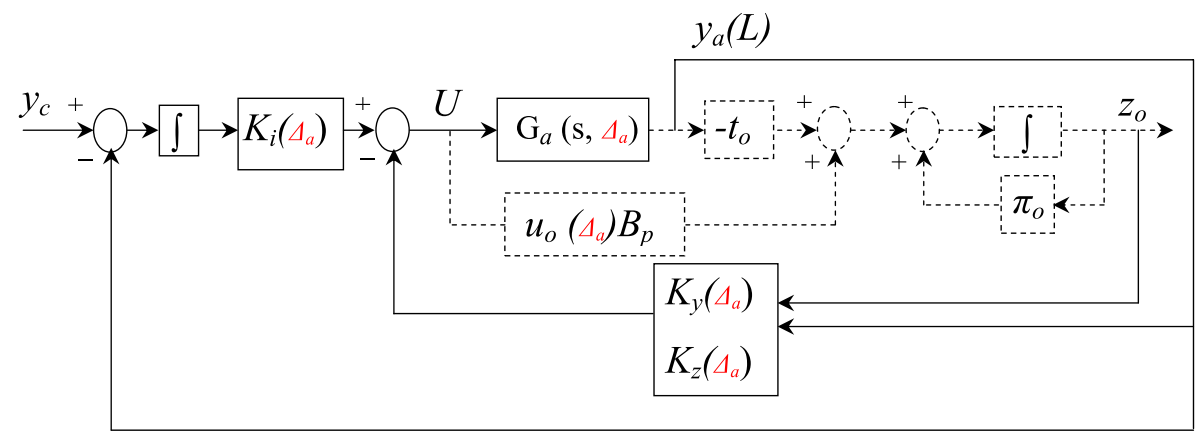

Fig. 8. Scheduled control scheme with the observer-based structure.

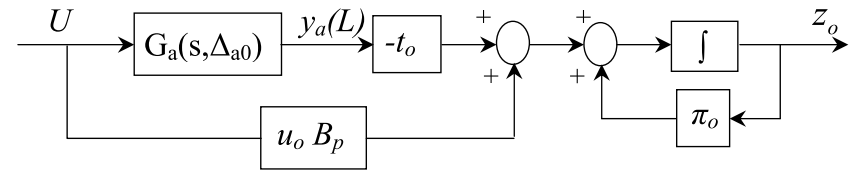

Fig. 9. Elementary observer of $z_{o}=u_{o} X_{p}$.

where $K_{c j}=\left[\begin{array}{lll}K_{y j} & K_{z j} & K_{i j}\end{array}\right]$, with $K_{y}=\left[\begin{array}{lll}K_{y 1} & K_{y 2} & K_{y 3}\end{array}\right]$, $K_{z}=\left[\begin{array}{llll}K_{z 1} & K_{z 2} & K_{z 3}\end{array}\right]$, and $K_{i}=\left[\begin{array}{lll}K_{i 1} & K_{i 2} & K_{i 3}\end{array}\right]$.

Let us now assume that $r$ triples have to be assigned for different configurations (at the same time) of the LPV system (12). Considering Lemma 1, the set $\left(v_{1}, \omega_{1}, \lambda_{1}\right) \cdots\left(v_{r}, \omega_{r}, \lambda_{r}\right)$ must satisfy the following conditions:

$$
\left\{\begin{array}{l}
\left(A_{p}\left(\Delta_{a 1}\right)-\lambda_{1} I_{n}\right) v_{1}-B_{p}\left(\Delta_{a 1}\right) \omega_{1}=0 \\
\vdots \\
\left(A_{p}\left(\Delta_{a r}\right)-\lambda_{r} I_{n}\right) v_{r}-B_{p}\left(\Delta_{a r}\right) \omega_{r}=0 .
\end{array}\right.
$$

Multimodel constraints are therefore defined by

$$
\left\{\begin{array}{c}
K_{c}\left(\Delta_{a 1}\right) C_{p}\left(\Delta_{a 1}\right) v_{1}=\omega_{1} \\
\vdots \\
K_{c}\left(\Delta_{a r}\right) C_{p}\left(\Delta_{a r}\right) v_{r}=\omega_{r} .
\end{array}\right.
$$

As such, considering the polynomial law (24), multimodel constraints (26) become

$$
\left\{\begin{array}{c}
\left(K_{c 0}+K_{c 1} \Delta_{a 1}+K_{c 2} \Delta_{a 1}^{2}\right) C_{p}\left(\Delta_{a 1}\right) v_{1}=\omega_{1} \\
\quad \vdots \\
\left(K_{c 0}+K_{c 1} \Delta_{a r}+K_{c 2} \Delta_{a r}^{2}\right) C_{p}\left(\Delta_{a r}\right) v_{r}=\omega_{r} .
\end{array}\right.
$$

This set of equations satisfies

$$
\begin{aligned}
{\left[\begin{array}{c}
K_{c 0}^{T} \\
K_{c 1}^{T} \\
K_{c 2}^{T}
\end{array}\right]^{T}=} & {\left[\begin{array}{l}
\omega_{1}^{T} \\
\vdots \\
\omega_{r}^{T}
\end{array}\right]^{T} } \\
& \times\left[\begin{array}{lll}
C_{p}\left(\Delta_{a 1}\right) v_{1} & \cdots & C_{p}\left(\Delta_{a r}\right) v_{r} \\
\Delta_{a 1} C_{p}\left(\Delta_{a 1}\right) v_{1} & \cdots & \Delta_{a r} C_{p}\left(\Delta_{a r}\right) v_{r} \\
\Delta_{a 1}^{2} C_{p}\left(\Delta_{a r}\right) v_{1} & \cdots & \Delta_{a r}^{2} C_{p}\left(\Delta_{a r}\right) v_{r}
\end{array}\right]^{-1} .
\end{aligned}
$$

The gains $K_{c 0}, K_{c 1}$ and $K_{c 2}$ are defined to satisfy the set of the $r$ multimodel constrains (26). More precisely, the gains allow assigning $3 \times r$ triples on $r$ LTI models from (12) in the entire set $\left[\begin{array}{ll}\Delta_{a m} & \Delta_{a M}\end{array}\right]$. If the $r$ LTI models correspond to worst case models, the multimodel synthesis leads to a scheduled controller that meet the robustness performance in the set $\left[\begin{array}{ll}\Delta_{a m} & \Delta_{a M}\end{array}\right]$.

To define the multimodel constraints, we have applied the following iterative procedure.

Procedure 1: Multimodel ESA.

\section{Step 1: Initialization:}

a) Design an output feedback controller (ESA methodology) with the observer considering a nominal model derived from (12). At this step, any frozen value of $\Delta_{a}$ can be used [this frozen value corresponds to $\Delta_{a 1}$ in (28)].

b) Identify the corresponding eigenstructure $\left(v_{1}, \omega_{1}\right)$.

Step 2: Analysis: Perform a worst case analysis (e.g., pole map or $\mu$-analysis) of the closed loop LTI models (operating points) in the parametric space $\left[\begin{array}{ll}\Delta_{a m} & \Delta_{a M}\end{array}\right]$. If the synthesis meets control specifications for all the selected LTI models, then stop. Otherwise identify a worst case model (or a model near the worst case model) $G_{w c}$ and continue with Step 3.

Step 3: Multimodel Synthesis: Improve the behavior of the worst case LTI model $G_{w c}$ identified at Step 2 respecting the closed loop specifications while preserving the properties of all the models treated before. It remains to assign the triple $\left(\lambda_{i+1}, v_{i+1}, \omega_{i+1}\right)$ on $G_{w c}$ simultaneously with the other assignments. Go back to Step 2.

This iterative procedure goes through the resolution of $r$ multimodel constraints (26) and the design of the output feedback gain (28). For the derivation of the scheduled gain, the matrix inversion in (28) must be possible and then it may be necessary to increase the degree of the polynomial (24) during the controller design procedure.

\section{Position Control of the Actuation Mechanism FT-G100}

The LPV model is used to define a set of 42 elementary LTI models in the space $\left[\begin{array}{ll}\Delta_{a m} & \Delta_{a M}\end{array}\right]$. The pole map of the open loop LPV system is shown in Fig. 10. Let us recall that in this paper we consider $\Delta_{a m}$ and $\Delta_{a M}$ as the operating points of the actuation mechanism corresponding to $V_{\text {in }}=5 \mathrm{~V}$ and $V_{\text {in }}=175 \mathrm{~V}$, respectively. From the set of the LTI models, the nominal model is selected such that the values of the stiffness $k_{a}(\cdot)$ and the damping $d_{a}(\cdot)$ are within 


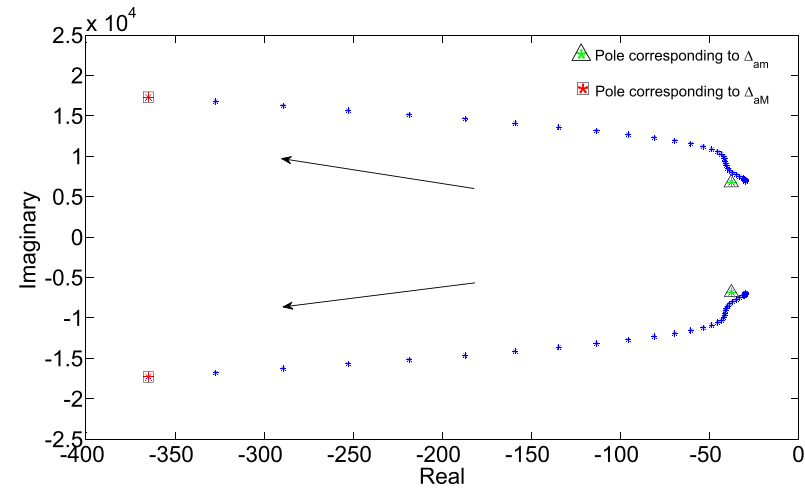

Fig. 10. Pole map of the open-loop LPV system. Arrows: evolution of the poles from $\Delta_{a m}$ to $\Delta_{a M}$.

the linear range [Fig. 5(b) and (c)]. For the nominal model $\Delta_{a 1}=\Delta_{a m}$.

The parameters of the observer are chosen such that $t_{o}=1$ and $\pi_{o}=-5 \times 10^{4}$. The parameter $\pi_{o}$ is related to the response time of the observer. It is chosen such that the observer is faster than the desired closed loop system.

Considering control specifications, three iterations of Procedure 1 have been required for the definition of the multimodel constraints.

Initialization 1: The ESA with the observer has been applied to the LTI model corresponding to $\Delta_{a 1}$ (nominal model).

1) Iteration 1: The worst case analysis [see the pole map in Fig. 11(a)] shows that control specifications are satisfied only for the nominal model. The complex-conjugate poles show that a set of LTI models have undamped vibrations. Moreover, the poles whose real value is close to zero (slow poles at the right side of $\lambda_{r 1}=-191$ ) show that the response time specification is not satisfied in the set $\left[\begin{array}{ll}\Delta_{a m} & \Delta_{a M}\end{array}\right]$. The pole map of the first iteration emphasizes that a single iteration is not sufficient to satisfy robust performances and justifies the need of a multimodel assignment. The worst case model is the one associated with $\Delta_{a 2}$ which corresponds to $V_{\text {in }}=175 \mathrm{~V}$. This model has a real pole (slowest pole of the pole map) and a complex-conjugate pole (poles with the highest complex value).

2) Iteration 2: The ESA with the observer has been applied to the LTI models corresponding to $\Delta_{a 1}$ and $\Delta_{a 2}$. The worst case analysis [see the pole map in Fig. 11(b)] shows that control specifications are satisfied for a set of elementary LTI models (including those with $\Delta_{a 1}$ and $\Delta_{a 2}$ ), but some models have vibrations that remain undamped. The worst case model is for $\Delta_{a 3}$ which corresponds to $V_{\text {in }}=45 \mathrm{~V}$.

3) Iteration 3: The ESA with the observer has been applied to the LTI models corresponding to $\Delta_{a 1}, \Delta_{a 2}$, and $\Delta_{a 3}$. The worst case analysis [see the pole map in Fig. 11(c)] shows that control specifications are satisfied for all the LTI models. The eigenstructure $\left(v_{1}, \omega_{1}\right),\left(v_{2}, \omega_{2}\right)$, and $\left(v_{3}, \omega_{3}\right)$ are related to the LTI models with $\Delta_{a 1}$, $\Delta_{a 2}$, and $\Delta_{a 3}$, respectively.
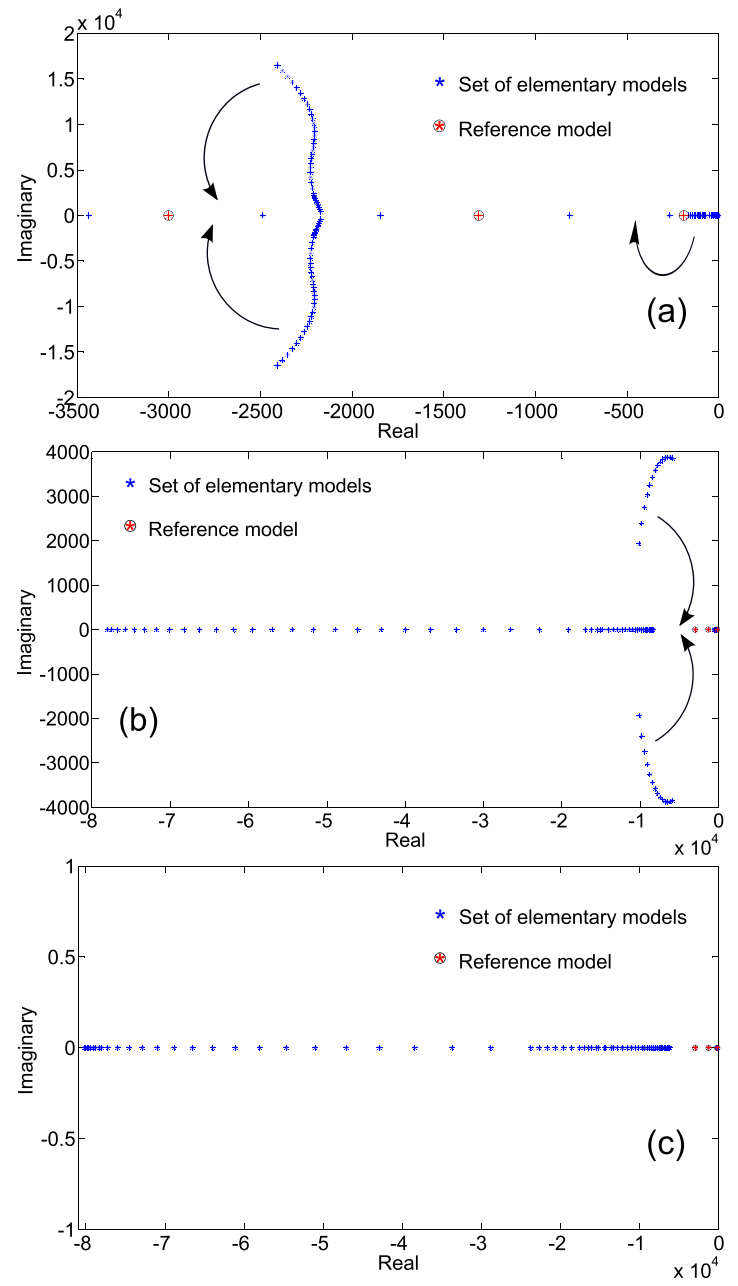

Fig. 11. Pole maps obtained during the iteration procedure. (a) First, (b) second, and (c) third iterations. Arrows show how the poles must be moved to satisfy the control specifications.

Finally, the gains $K_{c 0}, K_{c 1}$ and $K_{c 2}$ of the second-order polynomial (24) are defined through the relation (28) where $r=3$.

As a result, the gains $K_{c i}(i=1,2,3)$ are vectors of dimension 3 only. The resulting output feedback gain $K_{c}$ has consequently a very simple and low-dimensional structure which is very useful when the control law has to be implemented in simple microcontrollers (e.g., programmable integrated circuits). This is a real advantage compared with classical LPV controllers based on $H_{\infty}$ norm which often lead to high-order controllers and lead to implementation issues [21].

The relevance of the controller is demonstrated for a large (including the models used for the design) number of LTI models (Fig. 11). Because the controller is intended to be implemented on the nonlinear MEMS-based microgripper, its efficiency must also be assessed on the nonlinear model (11).

Comment: In the first iteration, the poles of the nominal model (i.e., with $\Delta_{a 1}$ ) are assigned to $\lambda_{1}=[-191-1.3 \times$ $\left.10^{3}-3 \times 10^{3}\right]$. In the second iteration, the poles of the models corresponding to $\Delta_{a 1}$ and $\Delta_{a 2}$ are assigned at the same time to $\lambda_{1}$ and $\lambda_{2}=\left[-248.27-1.7 \times 10^{4}-3.9 \times 10^{3}\right]$, respectively. In the third iteration, the poles of the models corresponding 

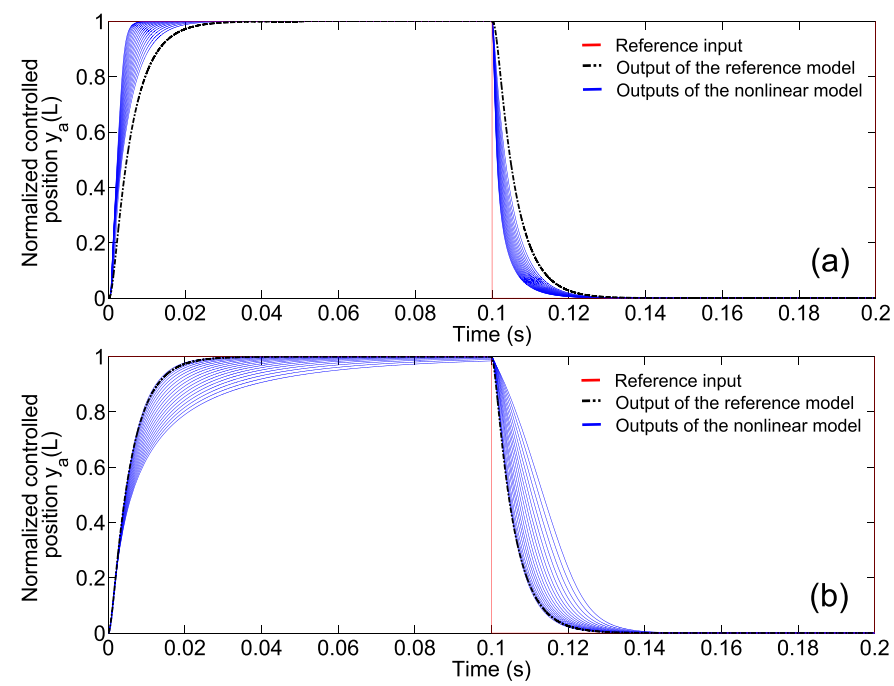

Fig. 12. Normalized step responses (simulation) of the controlled gripper arm at different operating points. (a) With the scheduled controller. (b) With the $\mathrm{LTI} / \mathrm{H} \infty$ controller.

to $\Delta_{a 1}, \Delta_{a 2}$ and $\Delta_{a 3}$ are assigned at the same time to $\lambda_{1}, \lambda_{2}$, and $\lambda_{3}=\left[-229.17-6.28 \times 10^{3}-2.88 \times 10^{4}\right]$, respectively.

\section{SimUlation VALIDATION AND EXPERIMENTAL IMPLEMENTATION}

\section{A. Simulation Validation}

For the robustness analysis, the gain-scheduled controller is applied in simulation on the nonlinear model (11). The position $y_{a}(L)$ is used as a feedback signal and also as scheduling variable for the controller.

To emphasis the importance of using a scheduled controller, a LTI/H $\infty$ controller is designed using the polynomial LPV model (12) for a frozen value $\Delta_{a}=\Delta_{a m}$. A weight function is used to apply a constraint to the sensitivity function of the closed loop system considering control specifications described beforehand.

Fig. 12(a) and (b) shows the normalized (to unity) controlled positions $y_{a}(L)$ for step references $y_{c}$ in the set [5 $\mu \mathrm{m} 90 \mu \mathrm{m}$ ]. Results are obtained with the scheduled controller [Fig. 12(a)] and with the LTI/H $\infty$ controller [Fig. 12(b)]. With the scheduled controller, the worst (i.e., highest) response time is $13.75 \mathrm{~ms}$, there is no overshoot and no static error. With the LTI/H $\infty$ controller, the worst response time is much bigger $(61.74 \mathrm{~ms})$, there is no overshoot, and the highest static error is equal to $0.02 \%$.

It is clear from Fig. 12 that the scheduled controller is essential to satisfy robust performance over a large operating range and especially in terms of closed loop response time. In this case, the proposed gain-scheduled control strategy gives satisfactory results.

\section{B. Experimental Implementation}

An experimental control system setup is used for the realtime implementation. It is composed of: 1) the FT-G100 microgripper; 2) the laser interferometer sensor (SP-120 SIOS Mebtechnik GmbH); 3) a controller board (dSPACE DS1103) with a real-time interface (RTI); and 4) an amplifier used to

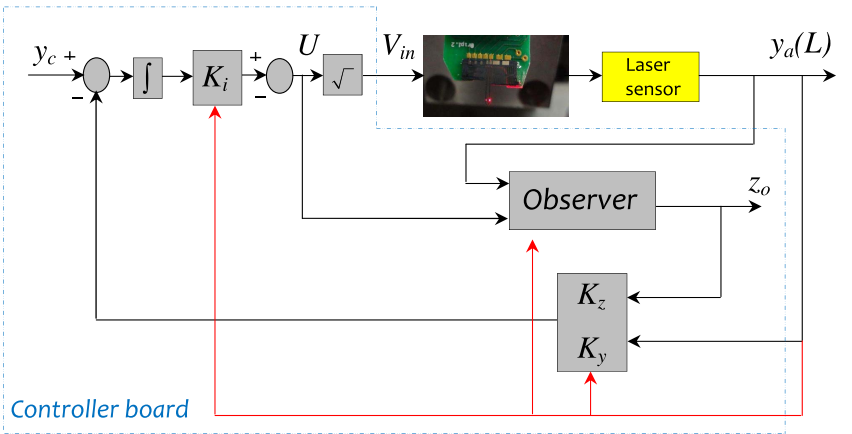

Fig. 13. Block diagram of the closed loop control system.

amplify the voltage signal at the output of the controller board which is limited to $10 \mathrm{~V}$.

The scheduled controller is designed through the MATLAB/Simulink software and is implemented into the controller board. The RTI allows applying various reference trajectories $y_{c}$. The laser spot of the interferometer is on the actuated arm and the signal provided by the sensor is taken into account in real-time by the controller board through a digital/digital interface. This signal is used both to schedule the controller and as a feedback signal. The square root of the controller output (i.e., $\sqrt{U}$ ) is applied to the comb drive actuator (Fig. 13).

An acquisition delay is generated by the digital/digital interface. The measurement delay is equal to four times the sampling time. Therefore, to reduce the effect of the delay on the controller performance, the sampling frequency has been set at $100 \mathrm{kHz}$. Moreover, to prevent any damage to the comb drive actuator, voltage saturation is applied such that the maximum actuation voltage that can be applied to the actuator is equal to $180 \mathrm{~V}$.

Experimental results are shown in Fig. 14 for different input references in the set of the operating range of the system. It is clearly shown that the wanted performance is obtained, that is, response time and overshoot. The response time of the closed loop system is lower than $10 \mathrm{~ms}$ in the overall operating range (let us recall that the response time in open loop is about $150 \mathrm{~ms}$ ). The mean static error in the operating range is equal to $0.26 \%$ which is due to vibrations of the actuated arm in frequencies higher than $2 \mathrm{kHz}$. Because the closed loop bandwidth of the system is equal to $100 \mathrm{~Hz}$, vibrations can be canceled with a low pass filter.

The controller allows obtaining a closed loop bandwidth of $100 \mathrm{~Hz}$ (the response time of the closed loop system is lower than $10 \mathrm{~ms}$ in the overall operating range) over an operating range of several tens of micrometers. To our best knowledge, such performance has never been achieved previously considering electrostatic MEMS-based microgrippers. Moreover, the controller has a very simple and lowdimensional structure. These results offer new perspectives for high-precision and high-speed manipulation tasks at the micrometer level. From the application point of view, one of the major interests of the controller is that it can improve the efficiency of micromanipulation tasks within a scanning electron microscope (SEM) (see examples in [4] and [31]). In such environments, the ability of implementing controllers 

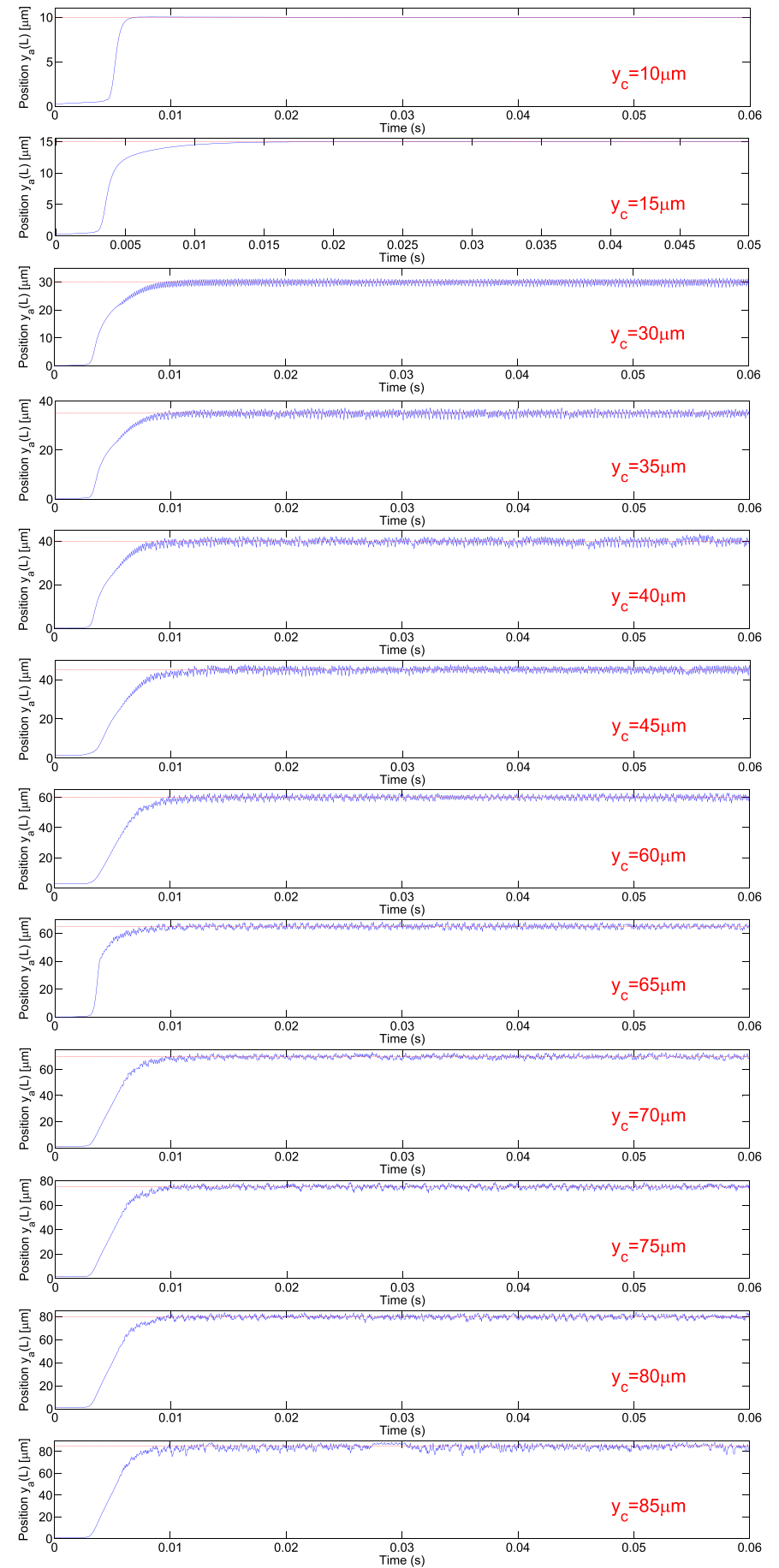

Fig. 14. Experimental closed loop responses of the actuation mechanism for different input references in the set of the operating range.

on a chip is of primary importance.

\section{Comparison With a PID Controller}

A Proportional Integral Derivative (PID) controller is designed and is implemented experimentally as a comparison. The nonlinear model (11) is used for the controller synthesis. The parameters of the controller are identified using ZieglerNichols method.

For an input reference $y_{c}=5 \mu \mathrm{m}$, the ultimate gain $K_{u}$ for which the output of the closed loop system oscillates with a constant amplitude is $K_{u}=8.91$. The period of such

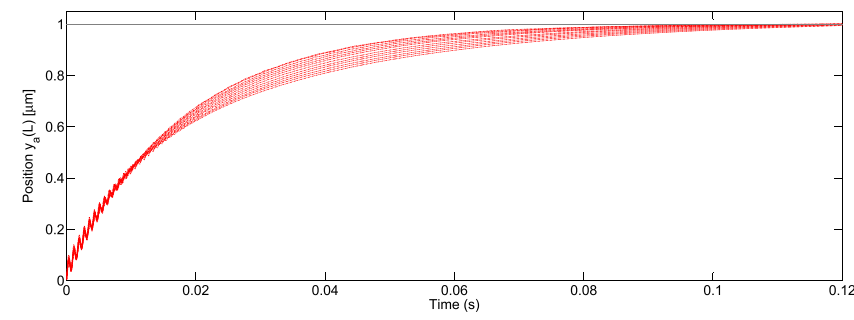

Fig. 15. Experimental normalized (to unity) step responses of the controlled gripping arm at different operating points (from 5 to $65 \mu \mathrm{m}$ ) with the PID controller.

oscillations is $T_{u}=0.9 \mathrm{~ms}$. Therefore, the proportional, the derivative, and the integral gains of the controller are, respectively, $K_{p}=5.34, K_{d}=1.12 \times 10^{-4}$, and $K_{i}=2.22 \times 10^{3}$. Fig. 15 shows the normalized (to unity) experimental controlled positions $y_{a}(L)$ for step references $y_{c}$ in the set [5 $\mu \mathrm{m} 65 \mu \mathrm{m}$ ]. With the PID controller, the worst response time (i.e., the highest) is $81.12 \mathrm{~ms}$ (more than eight times higher than the response time obtained with the scheduled controller). Note that, the two controllers (gain scheduling and PID) have not been implemented on the same FT-G100 microgripper. However, the static and dynamic properties of all FT-G100 microgripper series are closely the same.

\section{DISCUSSION ON REAL-TIME IMPLEMENTATION ISSUES}

The algorithm (multimodel and scheduled observer-based control strategy) has been implemented on the dSPACE board DS1103. The proposed control strategy has been implemented with a sampling frequency of $100 \mathrm{kHz}$ to reduce the effect of the measurement delay on the performance of the controller. It is nevertheless possible to do the implementation with a lower sampling frequency (for instance $20 \mathrm{kHz}$ ) provided that the measurement delay is considered during the controller synthesis. The delay can be added to the model of (12) by using a first-order Padé approximation. The order of the state space model is therefore increased by one. In this case, the controller can be implemented on a microcontroller at lower frequencies. The most important benefit of the proposed control strategy is its very simple structure (a second-order polynomial function to schedule an output feedback gain + a first-order scheduled observer) which do not require a significant computing time.

\section{CONCLUSION}

In this paper, we have proposed a systematic methodology for the modeling and the control of MEMS-based electrostatic microgrippers dedicated to embedded applications. The controller allows a very fast positioning of the gripping arm in a large operating range despite nonlinearities. Such performance are greatly needed for a range of microrobotics applications and have never been achieved previously with MEMS-based electrostatic microgrippers. To this end, a nonlinear dynamic model of the actuation mechanism has been proposed. First, it has been shown experimentally that the damping of the system is nonlinear and that the use of a third-order polynomial to describe the nonlinear stiffness of a comb drive actuator is limited. Therefore, both the stiffness and the damping have 
been described by polynomials of higher orders. To account the polynomial dependency of nonlinear parameters with respect to the position of the gripping arm, the nonlinear model has been reformulated into a polynomial LPV model and the controller has been designed through a gain-scheduled approach. To increase the number of degrees of freedom of the controller, a scheduled observer-based structure has been included in the control scheme. The structure of the gain feedback is polynomial whose order is defined a priori. This allows the user to set the dimension of the controller considering real-time implementation constraints. The relevance of the control approach is demonstrated through a robustness analysis and experimentally. Results show that a very wide (several tens of micrometers) and fast positioning of the gripping arm can be achieved with a very simple and low-dimensional (i.e., low-order) controller which is of a great importance from application point of view. This lead to new perspectives for high precision and fast micromanipulation tasks in embedded environments (e.g., SEM). Future applications will concern the use of the FT-G100 microgripper in closed loop for the fast characterization and assembly of carbon nanotubes by using a SEM which is nowadays one of the main issues and challenges in microrobotics.

\section{REFERENCES}

[1] N. Chaillet and S. Régnier, Microrobotics for Micromanipulation. New York, NY, USA: Wiley, 2010.

[2] C. Yamahata et al., "Silicon nanotweezers with adjustable and controllable gap for the manipulation and characterization of DNA molecules," in Proc. Int. Conf. Microtechnol. Med. Biol., Okinawa, Japan, May 2006, pp. 123-126.

[3] K. Kim, X. Liu, Y. Zhang, and Y. Sun, "Micronewton force-controlled manipulation of biomaterials using a monolithic MEMS microgripper with two-axis force feedback," in Proc. IEEE Int. Conf. Robot. Autom., Pasadena, CA, USA, May 2008, pp. 3100-3105.

[4] V. Eichhorn et al., "NanoLab: A nanorobotic system for automated pickand-place handling and characterization of CNTs," in Proc. IEEE Int. Conf. Robot. Autom., Kobe, Japan, May 2009, pp. 1826-1831.

[5] S. Muntwyler, B. E. Kratochvil, F. Beyeler, and B. J. Nelson, "Monolithically integrated two-axis microtensile tester for the mechanical characterization of microscopic samples," J. Microelectromech. Syst., vol. 19, no. 5, pp. 1223-1233, Oct. 2010.

[6] F. Beyeler et al., "Monolithically fabricated microgripper with integrated force sensor for manipulating microobjects and biological cells aligned in an ultrasonic field," J. Microelectromech. Syst., vol. 16, no. 1, pp. 7-15, Feb. 2007.

[7] A. P. Vijayasai, G. Sivakumar, M. Mulsow, S. Lacouture, A. Holness, and T. E. Dallas, "Haptic controlled three-axis MEMS gripper system," Rev. Sci. Instrum., vol. 81, no. 10, p. 105114, 2010.

[8] R. Legtenberg, A. W. Groeneveld, and M. Elwenspoek, "Comb-drive actuators for large displacements," J. Micromech. Microeng., vol. 6, no. 3, pp. 320-329, 1996.

[9] J. J. Abbott, Z. Nagy, F. Beyeler, and B. J. Nelson, "Robotics in the small, part I: Microbotics," IEEE Robot. Autom. Mag., vol. 14, no. 2, pp. 92-103, Jun. 2007.

[10] I. Kovacic and M. J. Brennan, The Duffing Equation: Nonlinear Oscillators and Their Behaviour. New York, NY, USA: Wiley, 2011.

[11] B. Ebrahimi and M. Bahrami, "Robust sliding-mode control of a MEMS optical switch," J. Phys., Conf. Ser., vol. 34, no. 34, pp. 728-733, 2006.

[12] K. B. Lee, A. P. Pisano, and L. Lin, "Nonlinear behaviors of a comb drive actuator under electrically induced tensile and compressive stresses," J. Micromech. Microeng., vol. 17, no. 3, p. 557, 2007.

[13] H. Alwi, A. Zolotas, C. Edwards, and K. Grigoriadis, "Sliding mode control design of an electrostatic microactuator using LPV schemes," in Proc. Amer. Control Conf., Montreal, QC, Canada, Jun. 2012, pp. $875-880$.

[14] A. Ferreira and S. S. Aphale, "A survey of modeling and control techniques for micro- and nanoelectromechanical systems," IEEE Trans. Syst., Man, Cybern. C, Appl. Rev., vol. 41, no. 3, pp. 350-364, May 2011.
[15] S. Khadraoui, M. Rakotondrabe, and P. Lutz, "Modeling and robust deflection control of piezoelectric microactuators modelled by zero-order numerator interval system," in Proc. 18th IFAC World Congr., Milan, Italy, 2011, pp. 9763-9768.

[16] J. M. Biannic, "Robust control of parameter varying systems: Aerospace applications," Ph.D. dissertation, Dept. DERA, Paul Sabatier Univ., Toulouse, France, 1996

[17] J.-F. Magni, "Multimodel eigenstructure assignment in flight-control design," Aerosp. Sci. Technol., vol. 3, no. 3, pp. 141-151, 1999.

[18] W. J. Rugh and J. S. Shamma, "Research on gain scheduling," Automatica, vol. 36, no. 10, pp. 1401-1425, 2000.

[19] D. J. Leith and W. E. Leithead, "Survey of gain-scheduling analysis and design," Int. J. Control, vol. 73, no. 11, pp. 1001-1025, 2000.

[20] P. Apkarian and P. Gahinet, "Convex characterization of gain-scheduled $\mathrm{H} \infty$ controllers," IEEE Trans. Autom. Control, vol. 40, no. 5, pp. 853-864, May 1995.

[21] C. Poussot-Vassal, O. Sename, L. Dugard, P. Gáspár, Z. Szabó, and J. Bokor, "A new semi-active suspension control strategy through LPV technique," Control Eng. Pract., vol. 16, no. 12, pp. 1519-1534, 2008.

[22] D. Robert, O. Sename, and D. Simon, "A reduced polytopic LPV synthesis for a sampling varying controller: Experimentation with a T inverted pendulum," in Proc. Eur. Control Conf., Kos, Greece, 2007.

[23] C. E. de Souza and A. Trofinon, "Gain-scheduled $\mathrm{H}_{2}$ controller synthesis for linear parameter varying systems via parameter-dependent Lyapunov functions," Int. J. Robust Nonlinear Control, vol. 16, no. 5, pp. 243-257, 2005.

[24] Y. Le Gorrec, J.-F. Magni, C. Doll, and C. Chiappa, "Modal multimodel control design approach applied to aircraft autopilot design," J. Guid., Control, Dyn., vol. 21, no. 1, pp. 77-83, 1998.

[25] M. Boudaoud, Y. Haddab, and Y. Le Gorrec, "Modeling and optimal force control of a nonlinear electrostatic microgripper," IEEE/ASME Trans. Mechatronics, vol. 18, no. 3, pp. 1130-1139, Jun. 2013.

[26] W. A. Moussa, H. Ahmed, W. Badawy, and M. Moussa, "Investigating the reliability of electrostatic comb-drive actuators used in micro fluidic and space systems using finite element analysis," Int. Can. J. Elect. Comput. Eng., vol. 27, pp. 195-200, Oct. 2002.

[27] W. Ye, S. Mukherjee, and N. C. MacDonald, "Optimal shape design of an electrostatic comb drive in microelectromechanical systems," J. Microelectromech. Syst., vol. 7, no. 1, pp. 16-26, Mar. 1998.

[28] J.-F. C. Magni, "An observer based multimodel control design approach," Int. J. Syst. Sci., vol. 30, no. 1, pp. 61-68, 1999.

[29] M. Boudaoud, Y. Le Gorrec, Y. Haddab, and P. Lutz, "Gain scheduled control strategies for a nonlinear electrostatic microgripper: Design and real time implementation," in Proc. IEEE 51st Annu. Conf. Decision Control, Maui, HI, USA, Dec. 2012, pp. 3127-3132.

[30] B. C. Moore and G. Klein, "Eigenvector selection in the linear regulator problem: Combining modal and optimal control," in Proc. IEEE Conf. Decision Control, Clearwater, FL, USA, Dec. 1976, pp. 214-215.

[31] V. Eichhorn, K. Carlson, K. N. Andersen, S. Fatikow, and P. Boggild, "Nanorobotic manipulation setup for pick-and-place handling and nondestructive characterization of carbon nanotubes," in Proc. IEEE/RSJ Int. Conf. Intell. Robots Syst., San Diego, CA, USA, Oct./Nov. 2007, pp. 291-296.

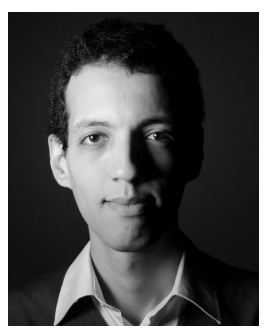

Mokrane Boudaoud received the Engineering degree in automatic control from the University of Science and Technology Houari Boumédiène, Algiers, Algeria, the M.S. degree in mechatronics and microrobotics from the University of FrancheComté, Besançon, France, in 2009, and the Ph.D. degree in control engineering from the Department of Automatic Control and Micro-Mechatronic Systems, Femto-ST Institute, in 2012.

His research interests included modeling and robust control of electrostatically actuated microelectromechanical systems-based microgrippers and noise characterization, modeling, and filtering for robotic micromanipulation. From 2013 to 2014 he was a Post-Doctoral Researcher with the Institut des Systèmes Intelligents et de Robotique. His field of interest was modeling and robust gripping force control at the microscale and trajectory planning of nanorobotic systems under a scanning electron microscope. Since 2014, he has been an Associate Professor with the Institut des Systèmes Intelligents et de Robotique, University Pierre et Marie Curie, Paris, France. 


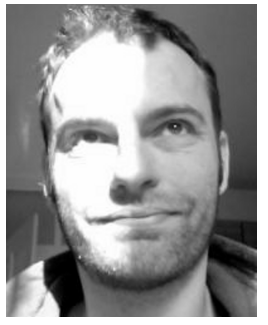

Yann Le Gorrec (M'13) was graduated as an Engineer in control, electronics, computer engineering at the National Institute of Applied Sciences, Toulouse, France, in 1995. He received the Ph.D. degree from the National Higher School of Aeronautics and Aerospace, Supaero, Toulouse, in 1998.

His field of interest was robust control and selfscheduled controller synthesis. From 1999 to 2008, he was an Associate Professor of Automatic Control with the Laboratory of Control and Chemical Engineering, Lyon Claude Bernard University, Villeurbanne, France. He worked on modeling of physicochemical processes, robust control, and modeling and control of distributed parameter systems. Since 2008, he has been a Professor with the National Engineering Institute in Mechanics and Microtechnologies, Besançon, France. His current field of research is control of smart material-based actuators, distributed microsystems, and more generally control of microactuators.

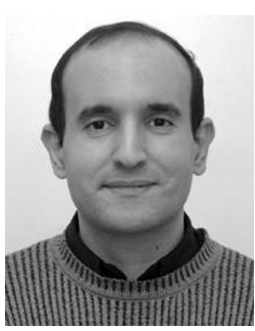

Yassine Haddab received the Engineering degree in electrical engineering from the University of TiziOuzou, Tizi Ouzou, Algeria, the M.S. degree from the National School of Mechanics and Microtechnology (ENSMM), Besançon, France, and the Ph.D. degree from the University of Franche-Comté, Besançon, in 2000.

His work dealt with the design, modeling, and control of micromanipulation systems. Since 2002, he has been an Associate Professor with ENSMM, where he teaches control theory, real-time control, and microrobotics. His research interests include the design and control of high-precision microrobots and microsystems. He also contributes to the development of new microrobots architectures.

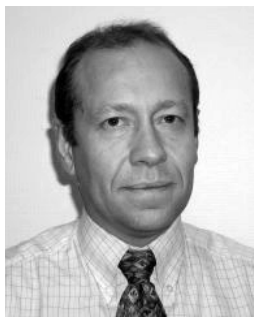

Philippe Lutz (M'07) received the Engineering degree from the National School of Mechanics and Microtechnology, Besançon, France, in 1990, and the Ph.D. degree in automation and computer science from the University of Franche-Comté, Besançon, in 1994.

He was an Associate Professor with the National Institute of Applied Sciences, Strasbourg, France, from 1994 to 2002. He joined the University of Franche-Comté, Besançon, as a Professor in 2002. He was the Head of the Research Group Automated Systems for Micromanipulation and Micro-Assembly of the Department of Automatic Control and Micro-Mechatronic Systems, FEMTO-ST Institute, from 2005 to 2011. He is currently the Director of the Ph.D. Graduate School of Engineering Science and Microsystems with more than $400 \mathrm{Ph} . \mathrm{D}$. students. His research activities are focused on the design and control of micromechatronic systems.

Prof. Lutz received several awards of IEEE, authored over 70 refereed publications (30 in high standard journals), serves as an Associate Editor of the IEEE TRANS ACTION ON AUTOMATION SCIENCE AND ENGINEERING, is a member of several Steering Committees, and is an active member of the IEEE Robotics and Automation Society Committee on Micro-Nano Robotics. 